\title{
Accumulation of Microfilaments in a Colonial Mutant of Neurospora crassa
}

\author{
Edward D. Allen, Robert J. Lowry, and Alfred S. Sussman \\ Veteran's Administration Hospital, Ann Arbor, Michigan 48105, and \\ Department of Botany, University of Michigan, Ann Arbor, Michigan 48104 \\ Received November 14, 1973, and in revised form February 21, 1974
}

\begin{abstract}
A morphological mutant of Neurospora crassa, snowflake, is shown to contain filaments which are about $70 \AA$ in diameter, and up to several microns long, and which usually bunch in groups of a few to several hundred. They may be found longitudinally or transversely arranged with respect to the long axis of the cell and, in many cases, they run up to the plasma membrane, but not through it. The filaments often are arranged in crystalline arrays but may also be found as separate filaments. Sometimes the filaments are closely appressed to nuclei and may be found inside them. It is likely that the filaments are not the result of the dissociation of microtubules and are most likely microfilaments like those found in other organisms. Their relationship to the origin of certain morphological mutants in Neurospora is discussed.
\end{abstract}

Cytoplasmic filaments ranging in size from $40-120 \AA$ in diameter and up to several microns long have been described in many organisms. It has been suggested that filaments in the smaller size range (50-70 $\AA$ ), generally referred to as microfilaments, are actinlike and involved in contractile processes.

Therefore, it has been hypothesized that microfilaments are involved in the following processes: cytoplasmic streaming in Acanthamoeba (19), Physarum $(7,13)$, Nitella (14), and higher plants $(15,16)$; cell elongation in Acytostelium (8), epidermal contraction in Amaroricium (4); and cleavage in jelly fish and polychaete worms (24), and squid cells (I). Other aspects of morphogenesis in animal cells also are believed in besuder the rontrol of microfilaments herause rutorhalasin $\mathrm{R}$ advug hat may

disrupt these, can arrest development when added to some systems (26). However, conflicting results have been obtained with this drug and its action on microfilaments has been questioned (9).

Despite the vast amount of literature on cytoplasmic filaments their fine structure has not been delineated in thin sections. Hatano and Oosawa (7) showed by negative staining that the F-actin they isolated from Physarum is composed of a twostranded helix. Similarly, negative staining of actinlike filaments, isolated from 
other organisms, also suggests such an arrangement $(13,18,19)$. When thin sections are used, the helical nature of the microfilaments has been derived from the beaded appearance of the microfilaments when viewed longitudinally. On the other hand, the filaments in transverse sections generally have been described as electron-dense dots, giving the impression that the filaments are solid cylinders.

In general, morphological mutants of Neurospora crassa are characterized by a very slow growth rate $(0.5 \mathrm{~mm} /$ hour $)$ and a higher frequency of branching as compared with wild-type strains. This results in colonies which have very compact mycelia and a mycelial front which advances very slowly. Some morphological mutants may not be as compact as others, but all differ appreciably from wild-type in linear growth rate of the colony, amount of aerial mycelium, compactness of aerial mycelium, and frequency of branching (12). The various mutants have been described mainly on the basis of the growth rates, a necessity imposed by lack of knowledge of the biochemical lesions of the mutants (6).

During the investigation of several morphological mutants of Neurospora crassa we found one which possesses large numbers of cytoplasmic filaments whose structure could be analyzed. This paper describes the appearance of these filaments in thin sections of fixed material.

\section{MATERIALS AND METHODS}

Organism. The morphological mutant of Neurospora crassa used for this study, strain 507 (snowflake), and the wild-type (standard strains), 4121a, and the Lindegren strain, were obtained through the courtesy of the Fungal Genetics Stock Center, Humboldt State College, Arcata, California.

Culture conditions. Cultures of Neurospora were grown in 500-ml Erlenmeyer flasks containing $200 \mathrm{ml}$ of distilled water with $2 \%$ sucrose and $2 \%$ of $50 \mathrm{X}$ Vogel's standard salts (25). Growth was initiated in flasks either by hyphal or conidial transfer.

Preparation for electron microscopy. Hyphae from liquid cultures were placed in vials containing fixatives at $0^{\circ} \mathrm{C}$ or room temperature. The fixative used most regularly was $3 \%$ glutaraldehyde in $0.1 \mathrm{M}$ sodium cacodylate $(\mathrm{pH} 7.4)$ at $0^{\circ} \mathrm{C}$ for 2 hours. Other fixatives included (a) $3 \%$ glutaraldehyde in $0.05 \mathrm{M}$ potassium phosphate $(\mathrm{pH} 7.2$ ) or (b) $1.5 \%$ of both glutaraldehyde and paraformaldehyde in $0.1 \mathrm{M}$ sodium cacodylate ( $\mathrm{pH} 7.4)$ or $0.05 \mathrm{M}$

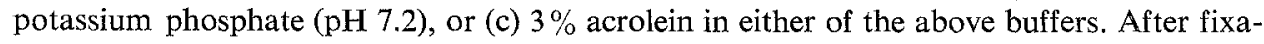
tion, the hyphae were washed several times and left in buffer overnight.

FIG. 1. Longitudinally oriented microfila ments in three cells. Arrows indicate groups of microfilaments. $\times 8600$.

FIG. 2. Transverse section of hyphae showing several groups of microfilaments in transverse and longitudinal view. Arrow at ribosomes $(R)$ indicates the small relative size of the microfilaments. $\times 49$ 800. Inset: higher magnif ication of region indicated by outline. Arrow indicates tubular appearance of one microfilament in transverse view. $\times 164000$.

FIG. 3. Three masses of microfilaments, one of which is perpendicular to septum $(S) . \times 16000$. 


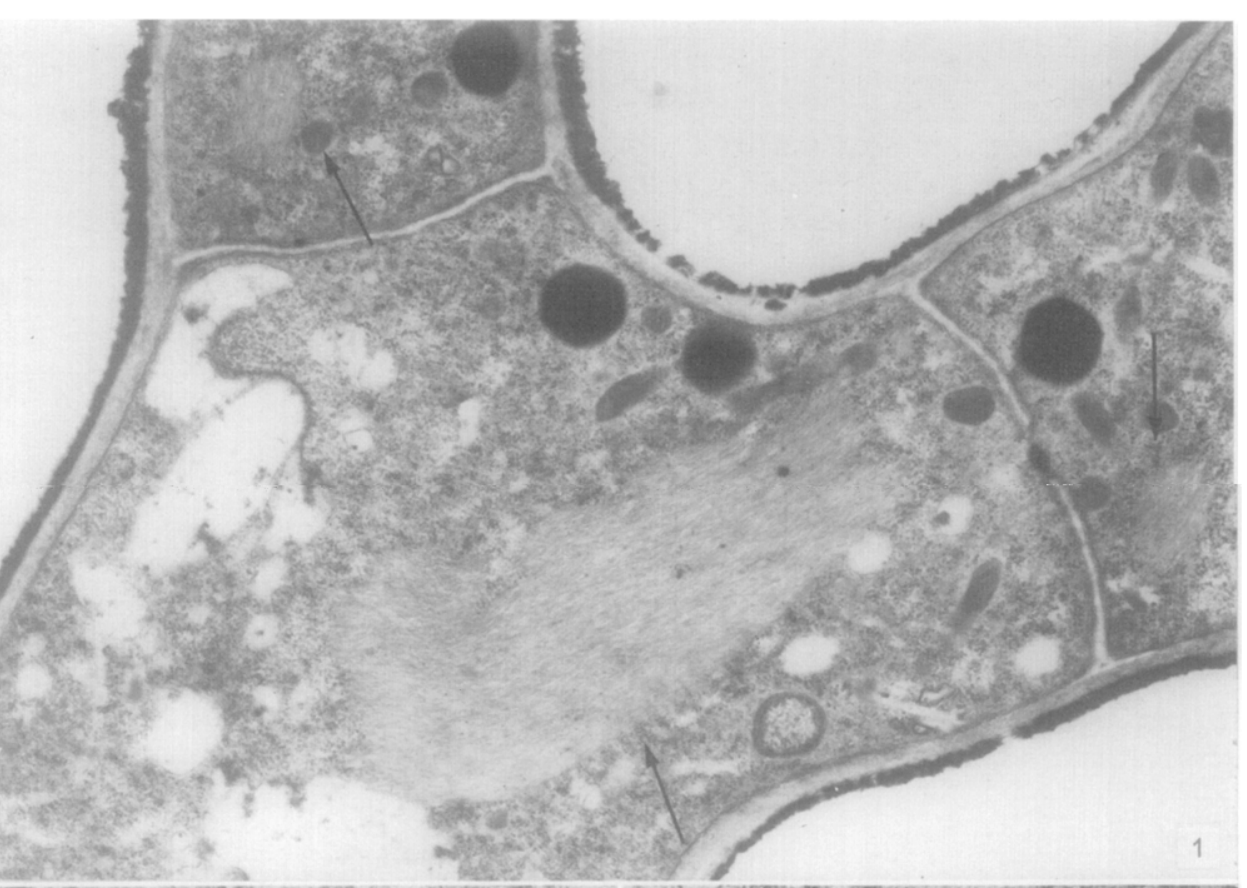

$\overline{\underline{ }}$
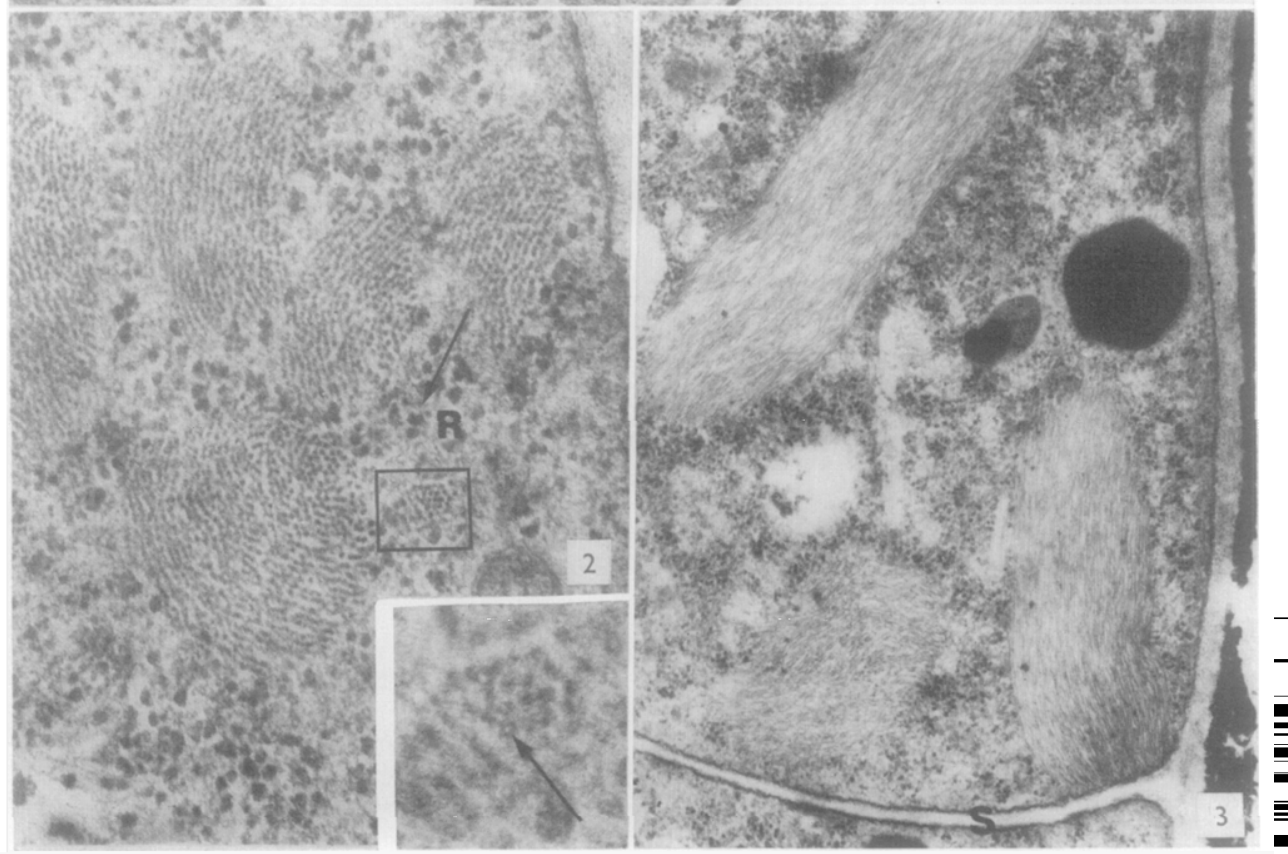
Postfixation $\left(1\right.$ hour at $0^{\circ} \mathrm{C}$ ) was carried out with the buffer used for fixation diluted $1: 1$ with aqueous $2 \%$ osmium tetroxide.

Dehydration in ethanol was followed by transfer to propylene oxide and embeddment in Epon [25 DDSA: 10 Epon 812:0.8 DMP-30 (23)].

Sections were cut with diamond or glass knives and stained with $2 \%$ aqueous uranyl acetate followed by lead citrate $(21)$. The sections were viewed with either Philips 300 , RCA-EMU4, or Hitachi 11 microscopes.

The microscope was calibrated with a carbon grating replica (28 800 lines per inch) obtained from E, F. Fullam Inc., Schenectady, New York. Microfilaments were measured on micrographs enlarged 4 times from negatives taken at $54000 \times$ at the time of calibration.

\section{RESULTS}

The growth pattern of snowflake (strain 507) is characteristic of many of the morphological mutants of Neurospora. Its hyphae radiate outward from a common center after hyphal or conidial transfer and grow at a rate of approximately $0.3 \mathrm{~mm}$ per hour compared to about $3.6 \mathrm{~mm}$ per hour for wild-type strains. The length of the cells of snowflake is about $100 \mu \mathrm{m}$ while that of the standard strains used is about $180-200 \mu \mathrm{m}$. The width of the cells of snowflake is about $20 \mu \mathrm{m}$ which is approximately one-half that of standard strains.

At the ultrastructural level, the main thing which distinguishes sections of snowflake from those of standard strains is the presence of large masses of a filamentous material. Unlike the wild-type strains, in which we have seen a few groups of filaments only in young vegetative hyphae, snowflake commonly contains masses of filaments in vegetative cells.

Figure 1 shows a section of three cells of snowflake, all of which possess the filamentous masses.

Filaments appear in groups ranging from a few to several hundred, and nearly all are found dispersed in the cytoplasm. They are not oriented in any particular direction with respect to the long axis of the cell; that is, they may be found in longitudinal or cross-sectional view when the cell is oriented longitudinally (Figs. 2 and 3). However, when extremely large masses of filaments are seen, they are usually oriented parallel to the longitudinal axis of the cell. The filaments are as common in apical cells as in those farther away from the growing tip. However, the extremely

FIG. 4. Group of microfilaments perpendicular to, but not passing through, the plasma membrane at cell wall $(W)$. The microfilaments appear granular near the plasma membrane. $\times 71000$.

FIG. 5. Appearance of microfilaments in hexagonal arrangement (arrows). The presence of longitudinally aligned filaments near the hexagonal ones suggests that some of the groups of microfilaments may be braided arrays. $\times 61600$.

FIG. 6. Longitudinal view of microfilaments in cytoplasm as well as microfilament-like structures in nucleus (arrow). Those found in nuclei appear the same as those observed in the cytoplasm. $\times 37000$. Frg. 7. Nucleus possessing microfilament-like structures (arrows) in transverse view. $\times 65000$. 

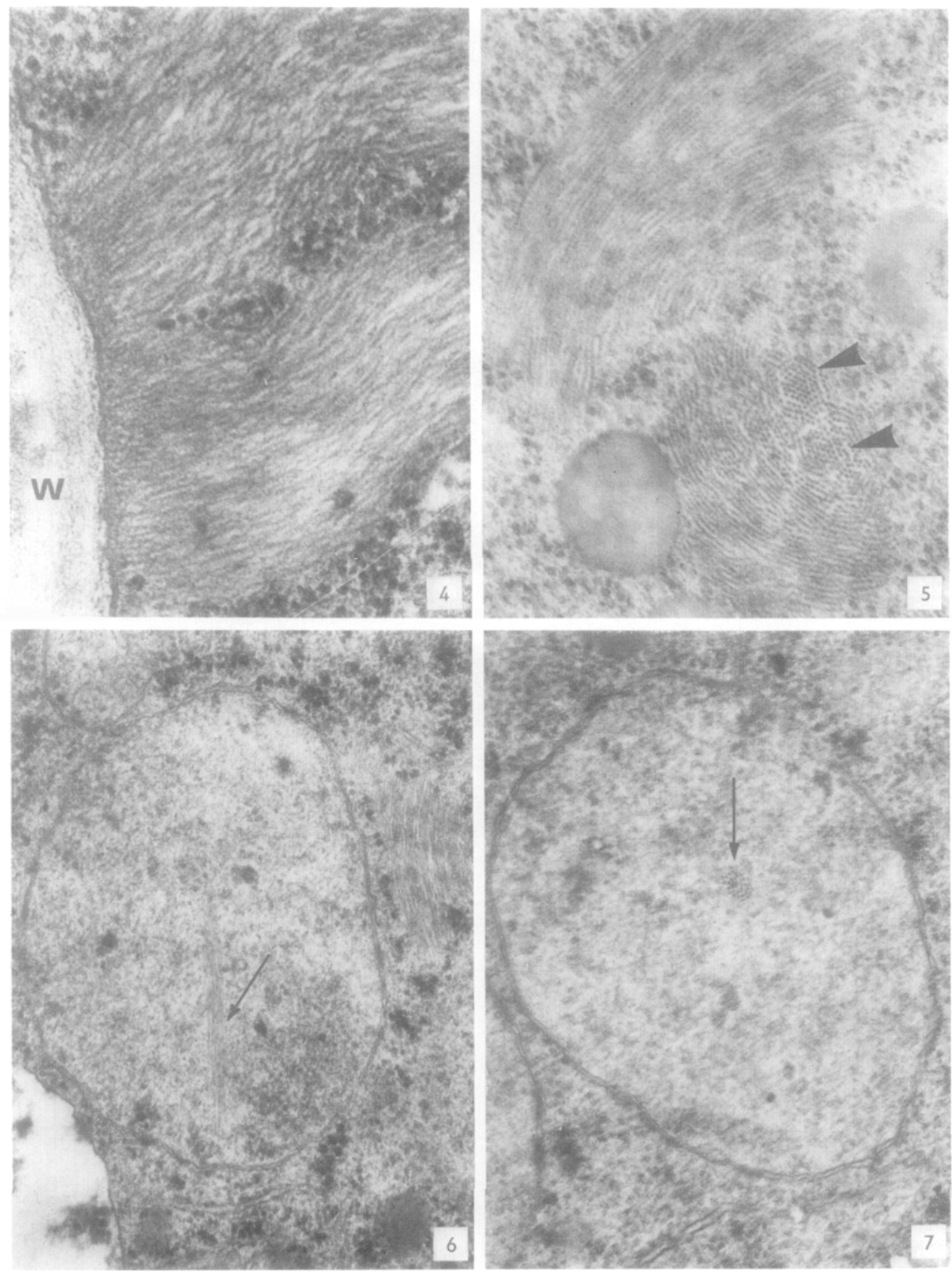
large masses appear more commonly in older cells. In many cases, longitudinal groups are seen to run up to the plasma membrane at the septum (Fig. 3) or wall (Fig. 4), but none have been seen to pass through it.

In longitudinal view, the individual filaments are approximately $70 \AA$ wide and up to several microns long. Their mean diameter is $68.8 \AA \pm 15 \AA$ and the range is from 40 to slightly above $100 \AA$. These values, and the standard deviation, were obtained from measurements of 111 filaments. Since the size of these filaments falls into the range of microfilaments, they will be described as such hereafter. Microfilaments are in close apposition and appear straight or slightly curved, and when in the former condition they present a somewhat crystalline appearance (Fig. 5). However, at other times they may appear loose and separated (Fig. 3).

In cross-sectional views the microfilaments appear as electron-dense dots approximately $70 \AA$ in diameter with up to several hundred grouped together in any one area. Occasionally, the microfilaments appear packed into hexagonal arrays (Fig. 5).

The only organelle in the cell with which the filaments seem to be associated is the nucleus. Several nuclei have been found to possess filamentous strands which are very similar in appearance to the microfilaments found in the cytoplasm (Fig. 6). The numbers seen in nuclei never approach those found in the cytoplasm. A single nucleus has been observed to possess about twenty of these strands in cross-sectional view (Fig. 7), which are similar in size to the microfilaments observed in the cytoplasm. We have not observed filaments in the nuclei of wild-type strains, but Beck et al. (2) have.

At higher magnifications, the usual appearance of cross sections of microfilaments is somewhat irregular and not distinctly circular. A few of the microfilaments at higher magnification appear to possess subunits (Figs. 8 and 9). The range of number of subunits in those microfilaments which show them is from two to four. The diameter of the subunits is approximately $25-35 \AA$.

A few of the microfilaments in cross section give the impression of being hollow tubes (Fig. 2, inset). The number of microfilaments which are tubular in cross-sectional view is very low compared to the number which show subunits, although the actual number of either is small when one considers the total number of microfilaments in any area. It should be mentioned that only extremely thin sections (darkgray to black) permitted the visualization of this subunit structure.

On viewing longitudinally oriented microfilaments at higher magnification, it appears that they are actually composed of more than one strand (Figs. 10 and 11).

As described in the section Materials and Methods, several different fixatives were used. Although they varied greatly in the effectiveness of preservation of background cytoplasm and various organelles, all showed the microfilaments to be the same size. Microtubules in the range 180-240 $\AA$ were never observed in the cyto- 

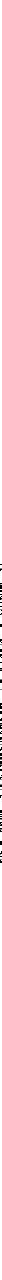
The presence of paraformaldehyde in the fixative containing glutaraldehyde preserves more information than the latter alone. This is evident in the retention of an electron dense outer cell wall (Fig. 1) and a denser mitochondrial matrix, neither of which are seen in cells only in glutaraldehyde.

\section{DISCUSSION}

The microfilaments just described have infrequently been observed by us in wildtype strains of Neurospora grown and prepared for electron microscopy under the same conditions used for snowflake. But several investigators have observed filaments in standard strains of Neurospora, including Beck et al. (2), who have shown that cultures obtained 4 hours after inoculation on media containing $2 \%$ sucrose or $15 \%$ glucose possess filaments ("striated inclusions"). These microfilaments, whose appearance is identical to those in snowflake, are $62 \AA$ in diameter and appear in the nucleus as well as the cytoplasm. The authors refer to the microfilaments as "rods," and they believe that individual elements are linked by fine filamentous strands and that the rods may be tubular.

As mentioned above, the individual microfilaments in snowflake do not appear to be rods, or solid cylinders. Instead, they appear to be composed of strands when observed in longitudinal sections, a conclusion that is supported by the subunits seen in cross-sectional view. We have not observed fine filamentous strands connecting microfilaments and feel that the tubular appearance of some microfilaments in cross-sectional view is related to their orientation. That is, osmium tetroxide used as a postfixative could contribute to the electron density of the strands, especially through thicker sections, thereby giving the impression of a continuous cylinder or a tubule, depending on whether the microfilaments are oriented in an exact transverse plane when sectioned.

Filaments in standard strains of Neurospora also have been reported by Wood and Luck (28), who found "paracrystalline inclusions" in a mitochondrial mutant (abnormal -1 ). These inclusions could be induced in standard strains by the addition of ethidium bromide or euflavine to the culture medium. The authors describe the filaments as being approximately $70 \AA$ wide and dotlike in cross section. After isolation and negative staining this material showed a banded pattern formed by units $86 \AA$ in length, arranged end-to-end. Wood and Luck found that the protein from paracrystalline inclusions is not a product of mitochondrial genes, and believe that it crystallizes as a result of a mitochondrial defect.

The variation in the diameter of Neurospora microfilaments (approximately 40$100 \AA$ ) could be caused by any of several factors related to fixation, orientation during sectioning, staining, etc. However, such variability could also be explained if 
microfilaments possessed different numbers of subunit strands. Pollard and Ito (17) noted that the smaller filaments in Amoeba range from 50 to $92 \AA$ in diameter. This size range might also indicate that variations in the number of subunit strands exist in Amoeba and might also imply that not all small filaments are like actin in forming a two-stranded helix. Variations in the number of strands in individual filaments could explain the large size variations which exist among many small filaments and could provide a mechanism to explain how larger filaments are formed, i.e., by increased numbers of subunit strands. Such a mechanism does not, however, explain the short $(0.5 \mu \mathrm{m})$ thick filaments in Amoeba (17) or the reason why heavy meromyosin binds only to the smaller filaments.

There is evidence that some of the microfilaments seen in electron micrographs are subunits of microtubules. Schultz and Case (22) have shown that neuronal microtubules are replaced by microfilaments in bicarbonate-buffered fixatives. On the other hand, fixatives containing cacodylate or phosphate buffers give normal microtubules. Microfilaments also have been seen after the use of mitotic inhibitors, such as colchicine, vinblastine sulfate, and podophyllotoxin, which are known to disrupt microtubules of the spindle apparatus (27). Consequently, we considered the possibility that the microfilaments observed in snowflake are artifacts of the kind discussed above. Glutaraldehyde when used in combination with osmium tetroxide has been shown to preserve microtubules. After using this fixation procedure as well as the others described in the Materials and Methods section, large numbers of microfilaments still were observed in snowflake and were never found assembled into microtubules.

Furthermore, where microtubules ( $250 \AA$ in diameter) have been observed in nuclei of snowflake, microfilaments of the usual kind still were found in the cytoplasm. Since nuclear microtubules were preserved it is unlikely that those occurring in the cytoplasm of the same cell would not be. Additionally, since microtubules have not been seen in the cytoplasm of Neurospora under fixation regimes that fix other organelles well, it seems unlikely that microfilaments are masses of microtubule subunits resulting from eoor fixation.

This study points to another hypothesis for the origin of certain morphological mutants of Neurospora. Such mutants are characterized, in comparison with standard strains, by the formation of dense colonies with a reduced rate of forward extension and an increased frequency of branching. One hypothesis is based upon the data of Tatum and collaborators $(5,10)$, which suggest that the walls of mutants contain significantly less glucose and more glucosamine than those of standard strains. In other mutants the primary effect has been traced to the formation of defective enzymes (3), or higher activites of some (11). That a mucopolysaccharide polymer may regulate the rate of growth by effects upon the plasma membrane has been proposed 30-741823 J. Ultrastructure Research 
in another case (20). Our finding of masses of microfilaments in snowflake suggests the additional possibility that the altered growth habit of this mutant is related to the accumulation of this material, perhaps because of effects upon one or more of the physiological processes in which microfilaments have been implicated.

\section{REFERENCES}

1. ARnold, J. M., J. Cell Biol. 41, 894 (1969).

2. Beck, D. P., Decker, G. L. and Greenwalt, J. W., J. Ultrastruct. Res. 33, 245 (1970).

3. Brody, S. and Tatum, E. L., Proc. Nat. Acad. Sci. U.S. 58, 923 (1967).

4. Cloney, R. A., J. Ultrastruct. Res. 14, 300 (1966).

5. DeTerra, N. and Tatum, E. L., Science 134, 1066 (1963).

6. GarnJobst, L. and TATUM, E. L., Genetics 57, 579 (1967).

7. Hatano, S. and Oosawa, F., J. Cell Physiol. 68, 197 (1966).

8. Hohl, H. R., Hamamoto, S. T. and Hemmes, D. E., Amer. J. Bot. 55, 783 (1968).

9. Holtzer, H. and SAnger, J. W., Develop. Biol. 27, 444 (1972).

10. Mahadevan, P. R. and Tatum, E. L., J. Bacteriol. 90, 1073 (1965).

11. Mahadevan, P. R. and Mahadkar, U. R., J. Bacteriol. 101, 941 (1970).

12. Murray, J. C. and Srb, A. M., Can. J. Bot. 40, 351 (1962).

13. Nachmias, V. T., HuXley, H. E. and Kessler, D., J. Mol. Biol. 50, 83 (1970).

14. NaGAI, R. and REBHUN, L. I., J. Ultrastruct. Res. 14, 571 (1966).

15. O'Brien, T. P. and Thimann, K. V., Proc. Nat. Acad. Sci. U.S. 56, 888 (1966).

16. Parthasarathy, M. V. and Mühlethaler, K., J. Ultrastruct. Res. 38, 46 (1972).

17. Pollard, T. D. and Ito, S., J. Cell Biol. 46, 267 (1970).

18. Pollard, T. D. and Korn, E. D., J. Cell Biol. 48, 216 (1971).

19. Pollard, T. D., Shelton, E., Weihing, R. R. and Korn, E. D., J. Mol. Biol. 50, 91 (1970).

20. Reissig, J. L. and Glasgow, J. E., J. Bacteriol. 106, 882 (1971).

21. Reynolds, E. S., J. Cell Biol 17, 208 (1963).

22. Schultz, R. L. and Case, N. M., J. Cell Biol. 38, 633 (1968).

23. Sporn, M. B., Wanko, T. and Dingman, W., J. Cell Biol. 15 , 109 (1962).

24. Szollosi, D., J. Cell Biol. 44, 192 (1970).

25. VoGel, H., Amer. Natur. 98, 435 (1964).

26. Wessells, N. K., Spooner, B. S., Ash, J. F., Bradley, M. O., Luduena, M. A., Taylor, E. L., WrenN, J. T. and Yamada, K. M., Science 171, 135 (1971).

27. Wisniewski, H., Shelanski, M. L. and Terry, R. D., J. Cell Biol. 38, 224 (1968).

28. Woon, D. D. and Luck, D. J. L., J. Cell Biol. 51, 249 (1971). 